\title{
Sirenomelia with major cardiac anomalies
}

\author{
RT Saggers, ${ }^{1}$ MMed (Paed); R Charlton, ${ }^{2}$ BHSc (Biomed); P Nongena, ${ }^{3}$ MDres (UK); S C Velaphi, ${ }^{2} \mathrm{PhD}$ \\ ${ }^{1}$ Department of Paediatrics and Child Health, Charlotte Maxeke Johannesburg Academic Hospital and Faculty of Health Sciences, \\ University of the Witwatersrand, Johannesburg, South Africa \\ ${ }^{2}$ Department of Paediatrics and Child Health, Chris Hani Baragwanath Academic Hospital and Faculty of Health Sciences, \\ University of the Witwatersrand, Johannesburg, South Africa \\ ${ }^{3}$ Department of Paediatrics and Child Health, New Somerset Hospital and Faculty of Health Sciences, University of Cape Town, \\ South Africa
}

Corresponding author: RT Saggers (robin.saggers@wits.ac.za)

\begin{abstract}
Sirenomelia is a rare and serious congenital anomaly characterised by fusion of the lower limbs, usually a single umbilical artery and malformations of the genitourinary and gastrointestinal tracts. In this report, we present clinical and radiological features, as well as autopsy findings, in a patient with sirenomelia diagnosed at the time of delivery. Major cardiac defects were observed, namely transposition of the great vessels and a hypoplastic left ventricle.
\end{abstract}

S Afr J Child Health 2021;15(4):224-225. https://doi.org/10.7196/SAJCH.2021.v15i4.1815

Sirenomelia is a congenital anomaly characterised by lower limb fusion associated with varying degrees of abnormalities of the genitourinary, cardiovascular, gastrointestinal and musculoskeletal systems. ${ }^{[1]}$ Although the cause of this defect is not known, the vascular steal hypothesis ${ }^{[2]}$ appears to be the most likely mechanism for its development.

The incidence of sirenomelia is reported to be about 1 per 100000 births, with a male-to-female ratio of $3: 1 .^{[1,2]}$ Its outcome is nearly always fatal, with death occurring in the perinatal period owing to renal tract abnormalities and associated complications. ${ }^{[3]}$ We present a patient with sirenomelia with cardiac abnormalities, which are not commonly reported in patients with this condition.

\section{Case presentation}

A 22-year-old primigravida mother, at 36 weeks' gestation, was referred from a local clinic for poor progress in labour. She attended antenatal care at the local clinic and her pregnancy was assessed to be normal and she had no history of diabetes mellitus. An antenatal ultrasound scan was not performed. She delivered via caesarean section for failed augmentation of labour.

The infant weighed $2700 \mathrm{~g}$ at birth, with Apgar scores of 3 at 1 minute and 1 at 5 minutes. On examination, there was a midline lower-limb tapering to a single digit-like structure with a single nail (Figs 1a and b). No cloacal, anal, genital or urethral opening was present and there was only a single umbilical artery. A fleshy skin-covered appendage was seen over the sacrum, but the spine felt normal on palpation.

\section{Case management}

Owing to the severe abnormalities seen, it was decided not to resuscitate the infant but offer supportive care instead; the infant subsequently demised. The parents were counselled about their infant's diagnosis and outcome. Death was attributed to severe congenital abnormalities.

\section{Investigations}

The X-rays of the bones showed sacral agenesis, a hypoplastic pelvis and a single distal limb resembling a femur (Fig. 2). A markedly hypoplastic common tibia was also observed. The bones of the upper limbs appeared normal.

On autopsy, the presence of a single umbilical artery was confirmed. The great vessels were transposed, with hyperplasia of the right ventricle and hypoplasia of the left ventricle. The lungs were hypoplastic, with the right lung showing three lobes and the left lung only a single lobe. The rectum ended blind and was distended by meconium. A large cystic mass was observed in the pelvis, containing urine and lined by large papillae, which proved to be a kidney on histological examination. Two small masses were found within the wall of the kidney and these were confirmed to be adrenal glands on histology. No genital organs were found within the abdomen. Chromosomal analysis of blood showed a 46XY karyotype.

\section{Discussion}

Sirenomelia, or mermaid syndrome, is a rare structural congenital abnormality characterised by fusion of the lower limbs, together with

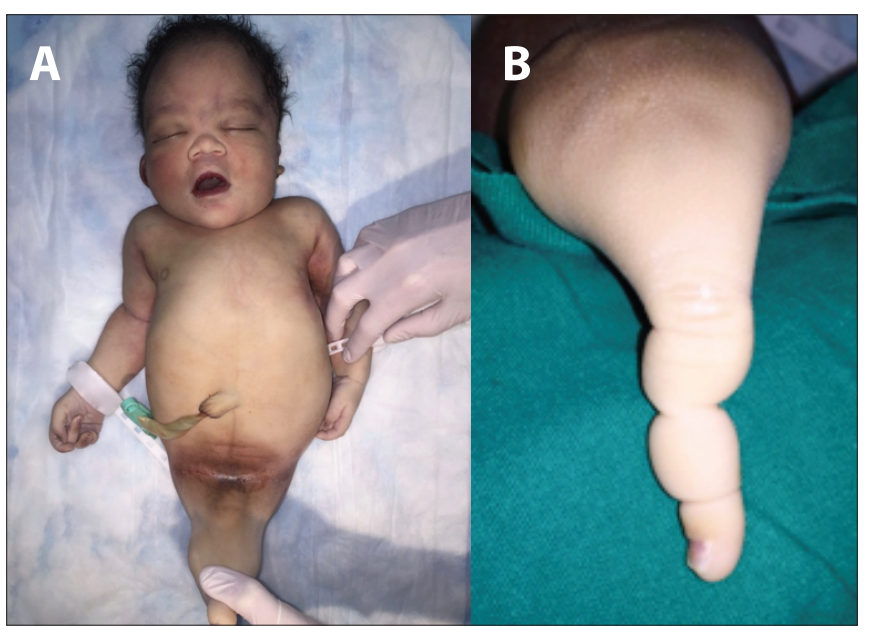

Fig. 1a. General appearance: bell-shaped chest, widely spaced nipples, single lower extremity, absence of external genitalia.

Fig. 1b. Finger-like appendage at distal end of single lower extremity. 


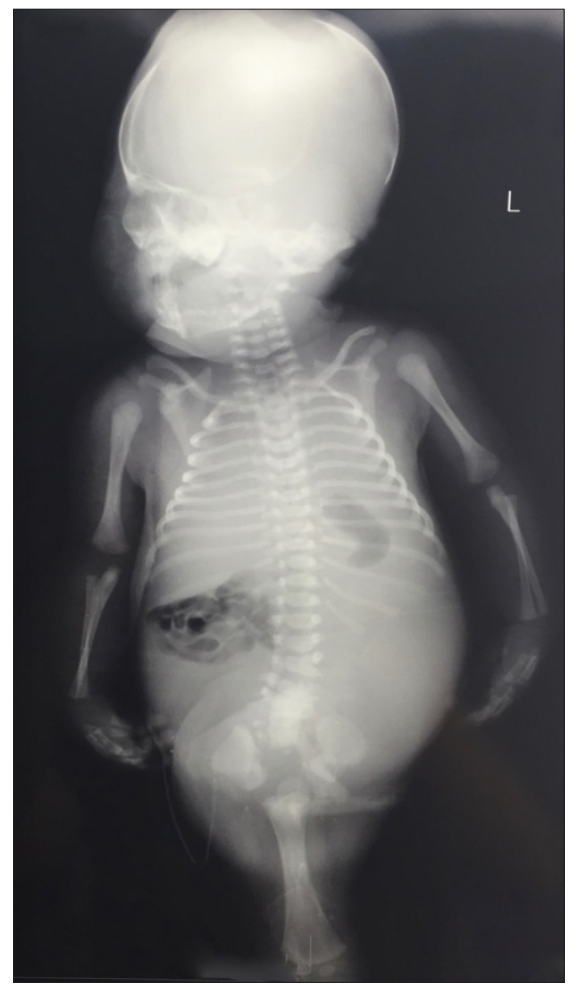

Fig. 2. Babygram (AP view): bell-shaped ribcage, rib-crowding, hypoplastic lungs, abnormal abdominal gas pattern, abnormal pelvis, normal upper limbs, single femur, absent tibia.

urogenital and anorectal malformations of varying degrees. Fatality usually results from complications of fetal renal tract abnormalities. Sirenomelia was first described in 1542 by Rocheas, and later also by Palfya in 1553 . The syndrome derived its name from this deformity resembling the single lower appendage of the mermaids, or 'sirens', of Greek mythology. ${ }^{[2]}$

Debate exists as to whether sirenomelia is a separate entity or if it represents a severe form of caudal regression syndrome. Evidence in favour of the former is that sirenomelia seems to be associated with a single umbilical artery, whereas this is not a finding characteristic of caudal regression syndrome. Furthermore, only $2 \%$ of sirenomelia cases have a history of maternal diabetes mellitus compared with $22 \%$ of cases described as representing caudal regression syndrome. ${ }^{[2]}$

The occurrence of a single umbilical artery has led to the pathogenesis of this syndrome being ascribed to the vascular steal hypothesis. The basic premise of this theory is that aberrant formation of the umbilical artery leads to persistence of the vitelline artery (type II single umbilical artery), which then assumes the role of both umbilical arteries. This single, large artery, or 'steal vessel', arises as a continuation of the abdominal aorta just distal to the diaphragm and diverts blood away from the caudal extremity of the embryo, towards the placenta. This results in deficient blood flow and nutrient supply to the caudal mesoderm, which, in turn, causes abnormal development or agenesis of caudal structures and the characteristic features of sirenomelia. ${ }^{[2]}$

Although the infant had a male genotype, no internal or external genital organs were noticed. The observation supports the vascular steal theory, as it points to disrupted blood supply to the gonads from the abdominal aorta below the origin of the steal vessel. ${ }^{[2]}$

The 'defective blastogenesis hypothesis' is an alternative theory for the pathogenesis of sirenomelia, proposing that abnormal development of the caudal mesodermal structures results from an embryological insult occurring at 28 - 32 days of gestation. Proposed examples of such an insult include maternal diabetes, pressure on the caudal extremity or maternal exposure to teratogens. ${ }^{[1,3]}$ In this case, no identifiable insults were noticed that could have resulted in abnormal development. Furthermore, the presence of a normal karyotype highlights that the pathogenesis of this case is unlikely to be related to a chromosomal abnormality but rather to a developmental or otherwise undetermined genetic abnormality.

The external morphological appearance of the lower limb fusion in sirenomelia encompasses a wide spectrum. Given the large number of possible morphologies, various classification systems have been proposed. The most widely accepted is that by Stocker and Heifetz. ${ }^{[4]}$ According to this classification system, this infant presented with type VII sirenomelia.

Although antenatal diagnosis of this condition is possible, resource constraints in public-sector healthcare settings in developing countries (such as South Africa) mean that appropriate antenatal ultrasound scans are performed only in high-risk pregnancies. As a result, there were no antenatal scans indicated for this pregnancy and the diagnosis was therefore made postnatally. Post-mortem examination was performed to confirm the diagnosis and assist with counselling of the parents.

In the literature, sirenomelia cases has rarely progressed to autopsy examination and consequently there are limited cases reporting cardiac abnormalities. ${ }^{[1,5,6]}$ None of these cases have reported transposed great vessels or a hypoplastic left ventricle. Neither of the mentioned theories on pathogenesis explains the cardiac anomalies, suggesting that these either are coincidental findings or due to a variant form of sirenomelia.

\section{Conclusion}

This paper presents a case of (type VII) sirenomelia ${ }^{[4]}$ with additional congenital abnormalities that have not been reported in the literature previously. This case report therefore adds to the body of literature, in that another variation in the abnormalities seen in this condition is described.

Acknowledgements. The authors thank the parents of the infant for consenting to publication of this case report and supporting photographs. We also thank Drs C Bailly and L Lambie (University of the Witwatersrand) for their input, and Drs C V Leisegang, E J van den Berg, M M Mokgoro and R M Maritz (National Health Laboratory Service) for the post-mortem and histology reports.

Author contributions. RTS and RC conceptualised the report, reviewed the literature and wrote the manuscript. PN and SCV supervised the study. All authors contributed to reviewing and revising earlier drafts of the report and approving the final manuscript.

Funding. None.

Conflicts of interest. None.

1. Nosrati A, Naghshvar F, Torabizadeh Z, Emadian O. Mermaid syndrome, Sirenomelia: A case report and review of literature. J Pediatr Rev 2013;1(1):64-69.

2. Fadhlaoui A, Khrouf M, Gaigi S, Zhioua F, Chaker A. The sirenomelia sequence: A case history. Clin Med Insights Case Rep 2010;3:41-49. https://doi. org/10.4137/CCRep.S5347

3. Samal SK, Rathod S. Sirenomelia: The mermaid syndrome: Report of two cases. J Nat Sci Biol Med 2015;6(1):264-266. https://doi.org/10.4103/09769668.149227

4. Stocker JT, Heifetz SA. Sirenomelia. A morphological study of 33 cases and review of the literature. Perspect Pediatr Pathol 1987;10:7-50.

5. Seidahmed MZ, Abdelbasit OB, Alhussein KA, Miqdad AM, Khalil MI, Salih MA. Sirenomelia and severe caudal regression syndrome. Saudi Med J 2014;35(Suppl 1):S36-S43.

6. Lynch S, Wright C. Sirenomelia, limb reduction defects, cardiovascular malformation, renal agenesis in an infant born to a diabetic mother. Clin Dysmorphol 1997;6(1):75-80.

Accepted 27 January 2021. 